\title{
Everybody is talking about Virtual Assistants, but how are people really using them?
}

\author{
Dr Marta Perez Garcia \\ Telefonica R\&D \\ Madrid, Spain \\ Marta.perezgarcia@telefonica.com Sarita.saffonlopez@telefonica.com \\ Sarita Saffon Lopez \\ Telefonica R\&D \\ Madrid, Spain
}

\author{
Hector Donis \\ Telefonica Digital \\ Madrid, Spain \\ Hector.Donis@telefonica.com
}

\begin{abstract}
Voice activated virtual assistants are growing rapidly in number, variety and visibility, driven by media coverage, corporate communications, and inclusion in a growing variety of devices. This trend can also be observed by how difficult it is becoming to find, among internet users, people who have not used or even heard of this new technology. Having said this, there is a visible shortage of academic research on this topic. Therefore, in the interest of creating a knowledge base around voice activated virtual assistants based on artificial intelligence, this multi-country exploratory research study was carried out. It concludes by providing information about the usage of four main voice activated virtual assistants (Siri, Google Assistant, Cortana and Alexa), comparisons between virtual assistant most frequently used and countries, and finally it establishes the base for further research activities.
\end{abstract}

Voice Activated Virtual Assistant, Artificial Intelligence, Technology adoption.

\section{INTRODUCTION}

Humans' interest in building a machine that is able to perform some kind of reasoning is not something new. In fact, we can go back to 300 B.C. to find Talos' story, a giant automaton made of bronze that protected the island of Crete. Dismissing tales, Ramon Lull explored the first traces of Artificial Intelligence (Al) with the mechanical calculator (calculus ratiocinator) around $1300 \mathrm{CE}$, which anticipated aspects of the Turing Machine and intended to make operations on concepts, not just numbers (Russell \& Norvig 2009, p. 16).

Several centuries later, Al research took form in the 1950s within a group of theorists and researchers, who predicted machines would become capable, in just a few decades, of carrying out any task humans can do (Simon, 1965; Minsky, 1967). However, it was until 1980s when Al research obtained the recognition it sought by launching the expert systems (Jackson, 1998), which simulated the decision-making ability of humans.

The 2010s arrived focusing on algorithms of machine learning by enabling computers to have access to large amounts of data, which comes back to what was expected in the 1950s (Samuel, 1959; Koza, 1996). This kind of application through a simplified interaction in games and hobbies is what enabled the adoption of $\mathrm{Al}$ at a user level. What is happening with Al implementation today on our daily basis then? One of many examples of our closest and most frequent interactions with it is the virtual personal assistants (Arafa and Mamdani, 2000).

Regardless of the wave of technology adoption with virtual assistants, little has been written in academia about it so that theory can be built upon. Most information regarding virtual assistants comes from brands websites or online magazines, but it is rather difficult to find theoretical foundations and solid hypothesis of users' behaviours around these technologies, beyond information such as "the most popular features" or "new functionalities". A necessity then is stressed to do formal research on their actual usage, differences among assistants, and how its use vary by users' age, sex or culture.

\section{VOICE ACTIVATED VIRTUAL ASSISTANTS}

Another problem encountered in the literature, is an absence of a common terminology: Al agents (Castelfranchi, 1998), virtual assistants (Martin and Allende, 2015), intelligent assistants (Kiseleva et al, 2016), and so on. What is important is that while the different terms are used, they are all referring to the same thing. For the purpose of this study, we will refer to them as Voice Activated Virtual Assistants (VAVAs).

For this study, we have gathered information of four key VAVAs: Siri (Apple), Google Assistant (Google), Cortana (Microsoft) and Alexa (Amazon). The reason behind this choice is that they have all been at least four years in the market, so they are more mature than other competitors, and, according to the 
statistic corporation Statista (201), Alexa and Google alone gather $87 \%$ of the global market share. Additionally, they use the same type of interaction with users, voice, and they are all talking Al databases. Also, they are accessible in affordable and widespread devices such as laptops, tablets, smartphones or own-brand devices, such as Amazon's Echo (which is different from the others, but we found interesting that Google and Apple are also moving towards this type of device, which could help give us a hint on future trends). These VAVAs are the Al play of four of the most powerful companies in the world, so it is vital to understand how its usage is being articulated.

\subsection{Siri}

Siri is Apple's VAVA, which was launched in 2011, the first of its kind, representing a competitive threat for competitors such as Google (Barnett, 2011), receiving both positive and negative reviews. For instance, some people found Siri was not able to understand and execute the tasks users were asking her to do, but they were also able to foresee its potential (Siegler, 2011). Currently, Siri can help users to set a reminder, an alarm, a calendar appointment, tell about the weather, take users to Google to find an answer, tell a joke, and so on.

\subsection{Google Assistant}

The days when Google was only a search engine have long passed. One of their most remarkable launches was Google Now in 2012, its intelligent personal assistant that uses natural language to provide a voice search. However, it did not engage in two-way conversations, which represented a drawback, so in May 2016 Google Assistant made its debut, as part of Google Allo, its messaging app. In 2017 Google announced a series of sneak peeks into the future by stating they are working on its assistant to support visual responses (LeFebvre, 2017), gather search sight information through the camera (Welch, 2017) or send money (Miller, 2017).

\subsection{Cortana}

In April 2013, at the BUILD developer conference, Microsoft introduced its VAVA Cortana, launched in January 2015. Microsoft defines Cortana as a personal digital assistant that is designed to help users carry out basic tasks as well as provide answers to users' questions. Cortana's focus is to work on devices with Microsoft OS, above all from the computer, but it is also available in Android smartphones. Cortana can schedule alarms or alerts, to send reminders based on user's location, or answer questions about traffic and sport scores.

\subsection{Alexa}

In November 2014, Amazon introduced Alexa and Amazon Echo in the US, but only available for Prime members. It officially launched in the US in 2015.
Alexa is the first voice activated virtual assistant linked to a stand-alone home device rather than integrated into existing electronic devices. UK and Germany receive Amazon Echo in September of that year, expanding the market in which they play in search of a major device adoption. Finally, in June 2017, Amazon Echo Show is launched in the US, a screen-device that multiplied the functionalities that Alexa could provide with a new interface. A key differentiator of Amazon strategy is the Alexa Skill Set, which enables anybody to design, build and help launch a new functionality in Alexa such as calling a cab or changing the colour of smart lights.

\section{METHODOLOGY AND SAMPLE}

Telefonica, the telecommunications multinational with its headquarters in Spain, is deeply involved in understanding and pursuing the vast opportunities that $\mathrm{Al}$ and VAVAs can bring to the company to deliver additional value and help create a new relationship with its customers. Therefore, the R\&D research team carried out this study as part of the exploration and establishment of a benchmark of VAVAs use across important markets in Telefonica's footprint.

A total of 3749 self-administered online questionnaires were sent during the first week of December 2017 to a panel of online users across seven countries: UK, Germany, Spain, Brazil, Argentina and Chile. Additionally, the US was included, not only as an assumed leader in VAVA adoption, but also to gather data on Alexa, which is not yet fully launched and established in several of the other markets studied. The survey was constructed by the research team and was composed of 11 multiple choice questions (excluding demographics) regarding subjects as knowledge and use of VAVAs, frequency of use, amount of tasks and place of use, among others.

The sample was distributed similarly in terms of gender (59\% female, $41 \%$ male) and included ages between 18 and $55(M=36.26, S D=10.45)$. All the respondents were active users of apps and internet through their mobile phones, and had diverse affinity with technology. For the descriptive and statistical analysis of VAVA's use, using SPSS platform, only the respondents that were active users were taken in account, resulting in a sample of 1047 users. Furthermore, in order to explore differences between the four main VAVAs, the study assured a sufficiently large sample per each of them.

\section{FINDINGS \& DISCUSSION}

\subsection{Knowledge and usage of VAVAs}

The first relevant result is that a great majority of online users in all countries of the study either uses or knows about VAVAs (Figure 1). Globally, just over half of online users say they know about VAVAs $(55.9 \%)$ and over a quarter of them say they use 
them $(27.9 \%)$. Only one in ten say that they have never heard of them (11.5\%) and a minority has used them in the past but stopped (4,6\%).

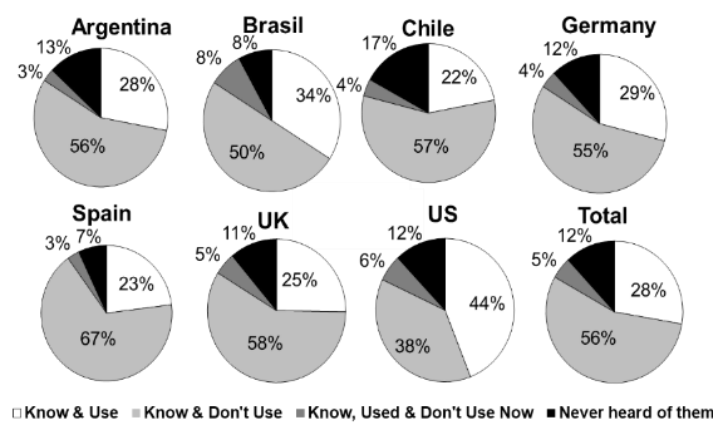

Figure 1: Distribution of knowledge and use of VAVAs in the seven countries of the study and total.

The research study has highlighted the fact that even if the first VAVA was launched several years ago, there is still more than two times the population that knows about VAVAs but does not use them than those who know and use them. As the data shows, VAVA adoption is not a question of knowledge about the service, as most non-users are aware of them, so it may be more related to a missing a trigger from knowledge to actual use. This is an area for continued research, to confirm not only the reasons for not using VAVAs from a user perspective, but also to understand what must happen in order to stimulate VAVA adoption to move from early adopters to early majority, and what can the industry do to make this easier.

Some important country differences exist, portrayed in Figure 1. As expected, the US is the country where most online users report using VAVAs $(44.2 \%)$ while Chile is at the other end of the spectrum with only $22 \%$ of VAVAs users and the highest percentage of people who has never heard of them $(17.1 \%)$. Spain is the country with the highest rate of people that are aware of the existence of VAVAs, but do not use them with $66.5 \%$ while the US has the lowest with $38.1 \%$.

These results confirm the hypothesis about the US being the most advanced market in the sample for VAVA adoption. In fact, the US is the only market where the number of VAVAs users is higher than those who know about but do not use them. Furthermore, considering this country is where Alexa is mostly present (24\% of its user base), it is possible to hypothesize that this VAVA, in the form of a standalone device, has enabled a further expansion of VAVAs into the early and late majority.

\subsection{Frequency of use and tasks requested}

The study has identified that current VAVA users are making use of it regularly (Figure 2); over $60 \%$ of the 1047 global users report using their VAVAs daily or several times a week (Almost daily). And, even though Chi-square test proves statistical significant the differences between counties for the frequency of use $(p<0,001)$; even the country that has the lowest percentage, Spain, still shows over half its users using it almost daily.

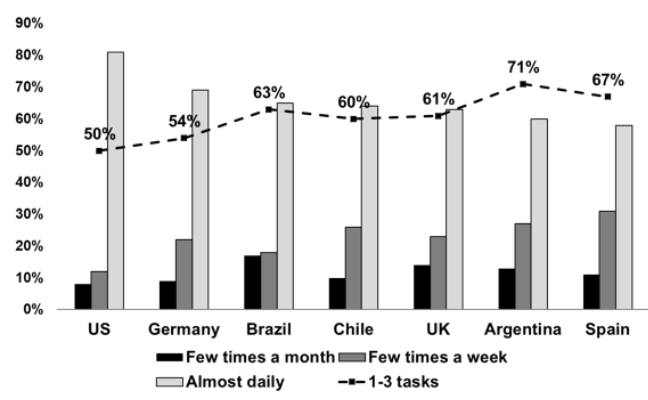

Figure 2: Frequency of use by countries and rate of user population that uses their VAVA for one to three tasks.

Nonetheless, it is also important to remark that the majority of VAVA users in each country only tend to carry out between one to three different tasks (or use cases such as setting up an alarm, asking what the weather is like, playing music, etc.). A possible explanation for the small number of tasks used could be that users just started using the VAVAs and have not had enough time to discover all of its use cases. However, this could be challenged by the fact that $55 \%$ reported using it for a year or longer.

Interestingly, Alexa is the VAVA that is being used for more tasks by its users (Figure 3 ). The number of users that execute six or seven tasks with Alexa, $17 \%$, almost triples that of other VAVAs users $(6 \%$ Google, 7\% Cortana and 8\% Siri). These results could be explored in a qualitative study to dive into the reason why more tasks are being used with Alexa, such as the fact that it is because it has more skills, or because the stand-alone device does not have to compete with the smartphone or computer other functionalities, or for its relationship with domestic tasks.

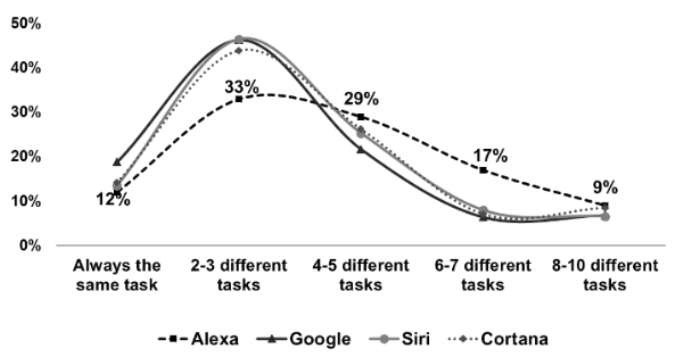

Figure 3: Tasks carried out by users with their VAVAs

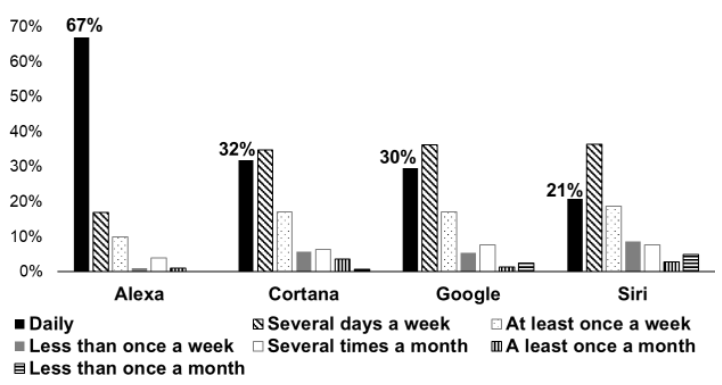

Figure 4: Frequency of use per VAVA. 
Another possible reason, hypothesised under the analysis performed for this study, could be a higher frequency of use of this VAVA. Alexa's users report using their VAVA daily more than twice, or even three times, as much as any other VAVA (Figure 4).

\subsection{Place and device of use}

Another relevant finding is that the use of VAVAs takes place mostly at home (Figure 5). In the case of Cortana, because of its link to Windows, people use it more at home than Siri or Google Assistant. However, even the latter two, with their significant mobile integration on smartphone devices, seem also to concentrate their use at home. Due to its Amazon Echo standalone device proposition, Alexa stands out even more for its use at home. Nonetheless, there is a small niche that declares to use it mostly or exclusively out of the home $(14 \%$ of Siri users and $8 \%$ of Google Assistant users).

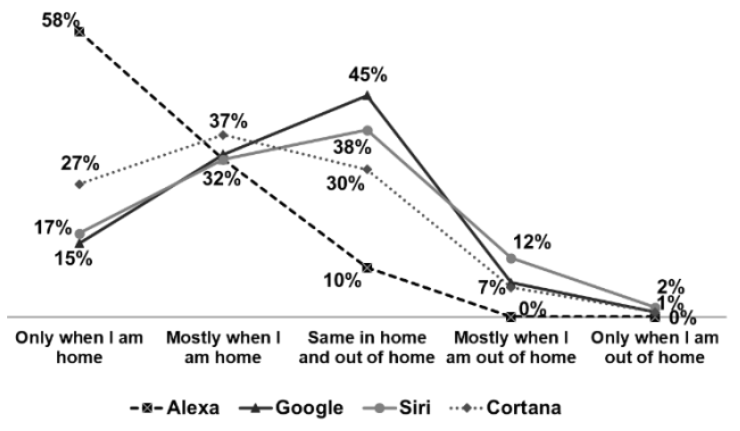

Figure 5: Place of use for the four major VAVAs

This study has also explored the most common device in which the VAVAs are used. The sample data shows that Siri and Google Assistant are mainly used in mobile devices (average globally of $75 \%$ and $73 \%$ of the time of use, respectively), while Cortana concentrates its main use on the PC with an average of $37 \%$ of the time of use being in this device. Alexa has the lion share of use on its own device with an average of $50 \%$, far superior and proven statistically significant by Chi-square test $(p<0.001)$, compared to the $3 \%$ to $6 \%$ average for the other VAVAs. None of these four VAVAs seems to dominate the tablet, which is the device where VAVAs are used the least, possibly due easier tactile screen interaction.

\subsection{Age comparisons}

In terms of generational differences in the use of VAVAs, four age-range groups were created (18 to 24,25 to 34,35 to 44 and 45 to 55 ). A key discovery was that the latter group is not only one that uses most frequently their VAVA, but the fact they use it for more tasks than the youngest group (Figure 6). While $34.9 \%$ of the 45 to $55 y$ rs reported using their VAVA daily, only $26.1 \%$ of the $18-24$ group did, and $6 \%$ reported using it less than once a month, being the highest of all four age groups. The differences between these two age groups was proven statistically significant ( $p$-value $=0.04$ ). Additionally, while $22.4 \%$ of the 45 to $55 y$ rs reported using their
VAVAs for more than six tasks, on average, only $12.8 \%$ of the younger groups did. These differences were also statically significant on a Chi-square test at a level of confidence of $99 \%$ ( $p$-value $<0.001$ ), and the contrast establishes the base for a further qualitative phase exploring the barriers of younger segments to increase the quantity of tasks as well as to determine the motivations of older segments for a more frequent and varied VAVAs usage.

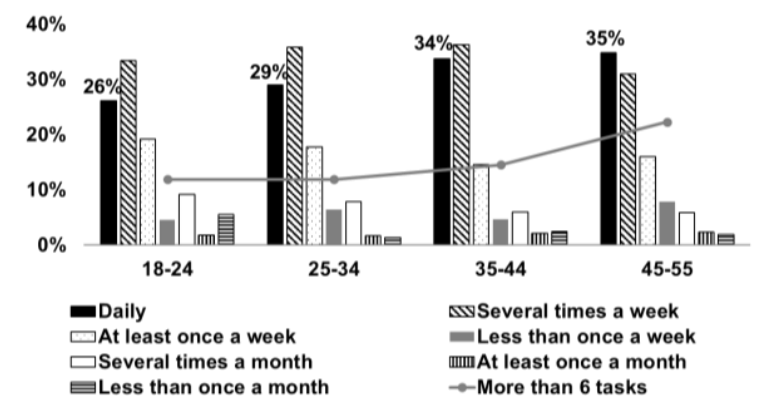

Figure 6: Frequency of use for the four age ranges, and rate of sample that uses VAVA for more than six tasks.

\section{CONCLUSIONS}

This study aimed to pursue a thorough understanding of current VAVA usage in seven different countries to establish a knowledge base from which more specific research studies will originate. The first finding of this study has been to establish that regardless young application of VAVAs, there is a high awareness across countries, even if its usage is unequal across countries.

This study has also identified that most people who start using this type of Al tend to make frequent use of it, which suggests the functionalities that they are able to carry out are valuable for users. In terms of age and use, this study has highlighted that, against what one might have thought, younger people are less attracted and are less heavy users than the oldest segment of the sample, both in frequency of use and number of tasks.

Finally, the study has also identified the key devices people typically use for interacting with their VAVAs. What draws attention to this finding is that although the smartphone is a rather common device to access the VAVA, most people use it more frequently at home, rather than when they are on the go, which it was expected and reported, mostly from Alexa, as it is linked to a home device.

\section{FUTURE RESEARCH OPPORTUNITIES}

This research study has triggered some remarkable aspects that are worth exploring separately. We are currently pursuing a multi-country study around personality attributes users perceive in these four VAVAs as well as identifying what would be the desired ones for the future. We are also exploring the current barriers for younger segments for not using VAVAs as frequently and for as many tasks as older segments. 


\section{REFERENCES}

Russell, Stuart J. and Norvig, P. (2009) Artificial Intelligence: A Modern Approach (3rd ed.). Prentice Hall, Upper Saddle River, New Jersey.

Russell, Stuart J. and Norvig, P. (2003) Artificial Intelligence: A Modern Approach (2nd ed.), Prentice Hall, Upper Saddle River, New Jersey.

Simon, H.A. (1965) The Shape of Automation for Men and Management. Harper \& Row, New York, New York.

Minsky, M. (1967) Computation: Finite and Infinite Machines. Prentice-Hall, Englewood Cliffs, New Jersey.

Jackson, P. (1998) Introduction to Expert Systems (3rd ed.). Addison-Wesley Longman Publishing Co., Boston, Massachusetts.

McCorduck, P. (2004) Machines Who Think (2nd ed.). A. K. Peters, Ltd., Natick, Massachusetts.

Samuel, A. (1959) Some Studies in Machine Learning Using the Game of Checkers. IBM Journal of Research and Development, 3(3), 211-229.

Koza, J.R., Bennett, F.H.. Andre, D. and Keane, M.A. (1996) Automated Design of Both the Topology and Sizing of Analog Electrical Circuits Using Genetic Programming. Artificial Intelligence in Design '96, 151-170.

Lally, A., Prager, J.M., McCord, M.C., Boguraev, B. K., Patwardhan, S., Fan, J. and Chu-Carroll, J. (2012) Question analysis: How Watson reads a clue. IBM Journal of Research and Development, 56(3.4), 2:1-2:14.

Zhang, Z. (2012) Microsoft kinect sensor and its effect. IEEE MultiMedia, 19(2), 4-10.

Chen, J. X. (2016) The evolution of computing: AlphaGo. Computing in Science \& Engineering, 18(4), 4-7.

Arafa, Y. and Mamdani, A. (2000) Virtual personal service assistants: towards real-time characters with artificial hearts. 5th International Conference on Intelligent User Interfaces, New Orleans, Louisiana, January 9-12, 2000, 9-12, ACM, New York, New York.

Castelfranchi, C. (1998) Modelling social action for Al agents. Artificial Intelligence, 103(1-2), 157-182.

Martín, P. J. and Allende, J. S. (2015) De Eliza a Siri: La evolución. Tecnología y desarrollo, 8, 3-30.

Kiseleva, J., Williams, K., Hassan Awadallah, A., Crook, A. C., Zitouni, I., and Anastasakos, T. (2016) Predicting user satisfaction with intelligent assistants. 39th International ACM SIGIR conference on Research and Development in Information Retrieval, Pisa, Italy, July 17-21, 2016, 45-54, ACM, New York, New York.

Siegler, M.G. (2011). The iPhone 4S: Faster, More Capable, And You Can Talk To It. TechCrunch. https://techcrunch.com/2011/10/11/iphone-4sreview/ (2018/02/15).

Barnett, E. (2011) Google's Eric Schmidt: Apple's Siri could pose 'threat'. http://www.telegraph.co.uk/technology/ericschmidt/8873664/Googles-Eric-Schmidt-ApplesSiri-could-pose-threat.html (2018/02/15).

O'Kane, S. (2016) Siri's big upgrades won't matter if it can't understand its users. https://www.theverge.com/2016/6/14/11925076/s iri-apple-wwdc-ios-updates-voice-recognition (2018/02/15).

Kahn, J. (2017) Apple engineers share behind-thescenes evolution of Siri \& more on Apple Machine Learning Journal. https://9to5mac.com/2017/08/23/evolution-sirimachine-learning-journal/ (2018/01/22).

LeFebvre, R. (2017) Google Assistant now accepts typed and verbal cues. https://www.engadget.com/2017/05/17/googleassistant-now-accepts-typed-and-verbal-cues/ (2018/02/18).

Welch, C. (2017) Google Assistant will soon search by sight with your smartphone camera. https://www.theverge.com/2017/5/17/15648128/g oogle-assistant-camera-sight-search-feature-io2017 (2018/02/18).

Miller, P. (2017) You'll soon be able to send money with Google Assistant. https://www.theverge.com/2017/5/18/15660728/g oogle-assistant-payment-api-peer-to-peermoney-money-money-io-2017 (2018/02/18).

Pega Systems Inc. (2017) What Consumers Really Think About Al: A Global Study. Retreived May 18, 2018 from https://www.pega.com/ai-survey

Statista (2017) Worldwide intelligent/digital assistant market share in 2017 and 2020, by product. Retreived May 18, 20118 from https://www.statista.com/statistics/789633/worldw ide-digital-assistant-market-share/ 